\title{
Teaching Video NeuroImages: Cautious walking gait in siblings with hereditary hyperekplexia
}

Chao Zhang, MM,* Shi-Ge Wang, MM,* Yan Wang, MM, Xiao-Li Liu, MM, and Li Cao, MD, PhD

Neurology ${ }^{\circledR}$ 2019;92:e2068-e2069. doi:10.1212/WNL.0000000000007375

A 48-year-old man and his 41-year-old sister complained of pathogenic startles since birth. They carried a homozygous deletion mutation in GLRA1: c.754delC (p. L252X). They felt stiff in 4 limbs with expressionless face when they were alarmed by unexpected touch and sound stimulation, startled, and fell down with stiffed limbs since walking independently. Therefore they adopted a cautious walking gait as a subconscious self-protection (videos 1 and 2 ).

With clonazepam treatment, pathologic cautious walking gait can be dramatically improved. Cautious walking gait is an interesting and easily observable manifestation of hereditary hyperekplexia. ${ }^{1,2}$

\section{Disclosure}

C. Zhang, S. Wang, and Y. Wang report no disclosures relevant to the manuscript. X. Liu is in charge of Shanghai Municipal Commission of Health and Family Planning (20184Y0056). L. Cao is in charge of National Natural Science Foundation of China (81571086 and 81870889), Shanghai Municipal Education Commission-Gaofeng Clinical Medicine Grant (20161401), and Interdisciplinary Project of Shanghai Jiao Tong University (YG2016MS64). Go to Neurology.org/N for full disclosures.

\section{Study funding}

This project is supported by National Natural Science Foundation of China (81571086 and 81870889), Shanghai Municipal Education Commission-Gaofeng Clinical Medicine Grant Support (20161401), Shanghai Municipal Commission of Health and Family Planning (20184Y0056), and Interdisciplinary Project of Shanghai Jiao Tong University (YG2016MS64).

\author{
Correspondence \\ Dr. Cao \\ caoli2000@yeah.net \\ or Dr. Liu \\ liuxl@rjlab.cn
}

\section{MORE ONLINE}

Videos

$\rightarrow$ Teaching slides

links.lww.com/WNL/

A894

*These authors contributed equally to this work.

From the Department of Neurology (C.Z., S.-G.W., Y.W., X.-L.L., L.C.), Rui Jin Hospital \& Rui Jin Hospital North, Shanghai Jiao Tong University School of Medicine; and Department of Neurology (X.-L.L.), Shanghai Fengxian District Central Hospital, Shanghai Jiao Tong University Affiliated Sixth People's Hospital South Campus, Shanghai, China. 


\section{Appendix Authors}

\begin{tabular}{|c|c|c|c|}
\hline Name & Location & Role & Contribution \\
\hline $\begin{array}{l}\text { Chao } \\
\text { Zhang, } \\
\text { MM }\end{array}$ & $\begin{array}{l}\text { Department of } \\
\text { Neurology, Rui Jin } \\
\text { Hospital and Rui Jin } \\
\text { Hospital North, } \\
\text { Shanghai Jiao Tong } \\
\text { University School of } \\
\text { Medicine }\end{array}$ & Author & $\begin{array}{l}\text { Data collection and } \\
\text { evaluation, video } \\
\text { shooting, drafting } \\
\text { the manuscript }\end{array}$ \\
\hline $\begin{array}{l}\text { Shi-Ge } \\
\text { Wang, } \\
\text { MM }\end{array}$ & $\begin{array}{l}\text { Department of } \\
\text { Neurology, Rui Jin } \\
\text { Hospital and Rui Jin } \\
\text { Hospital North, } \\
\text { Shanghai Jiao Tong } \\
\text { University School of } \\
\text { Medicine }\end{array}$ & Author & $\begin{array}{l}\text { Image editing, } \\
\text { manuscript drafting } \\
\text { and revision }\end{array}$ \\
\hline $\begin{array}{l}\text { Yan } \\
\text { Wang, } \\
\text { MM }\end{array}$ & $\begin{array}{l}\text { Department of } \\
\text { Neurology, Rui Jin } \\
\text { Hospital and Rui Jin } \\
\text { Hospital North, } \\
\text { Shanghai Jiao Tong } \\
\text { University School of } \\
\text { Medicine }\end{array}$ & Author & Data evaluation \\
\hline $\begin{array}{l}\text { Xiao-Li } \\
\text { Liu, } \\
\text { MM }\end{array}$ & $\begin{array}{l}\text { Department of } \\
\text { Neurology, } \\
\text { Shanghai Fengxian } \\
\text { District Central } \\
\text { Hospital, Shanghai } \\
\text { Jiao Tong University } \\
\text { Affiliated Sixth } \\
\text { People's Hospital } \\
\text { South Campus }\end{array}$ & $\begin{array}{l}\text { Corresponding } \\
\text { author }\end{array}$ & $\begin{array}{l}\text { Data evaluation, } \\
\text { manuscript revision }\end{array}$ \\
\hline $\begin{array}{l}\text { Li Cao, } \\
\text { MD, } \\
\text { PhD }\end{array}$ & $\begin{array}{l}\text { Department of } \\
\text { Neurology, Rui Jin } \\
\text { Hospital and Rui Jin } \\
\text { Hospital North, } \\
\text { Shanghai Jiao Tong } \\
\text { University School of } \\
\text { Medicine }\end{array}$ & $\begin{array}{l}\text { Corresponding } \\
\text { author }\end{array}$ & $\begin{array}{l}\text { Funding, data } \\
\text { collection and } \\
\text { evaluation, study } \\
\text { design and } \\
\text { supervision, } \\
\text { manuscript } \\
\text { revision, final } \\
\text { approval }\end{array}$ \\
\hline
\end{tabular}

\section{References}

1. Huang Z, Lian Y, Xu H, Zhang H. Weird laughing in hyperekplexia: a new phenotype associated with a novel mutation in the GLRA1 gene? Seizure 2018;58:6-8.

2. Bode A, Lynch JW. The impact of human hyperekplexia mutations on glycine receptor structure and function. Mol Brain 2014;7:2. 


\section{Neurology}

\section{Teaching Video NeuroImages: Cautious walking gait in siblings with hereditary hyperekplexia \\ Chao Zhang, Shi-Ge Wang, Yan Wang, et al. \\ Neurology 2019;92;e2068-e2069 \\ DOI 10.1212/WNL.0000000000007375}

This information is current as of April 22, 2019

\section{Updated Information \& Services}

References

Subspecialty Collections

Permissions \& Licensing

Reprints including high resolution figures, can be found at: http://n.neurology.org/content/92/17/e2068.full

This article cites 2 articles, 0 of which you can access for free at: http://n.neurology.org/content/92/17/e2068.full\#ref-list-1

This article, along with others on similar topics, appears in the following collection(s):

All Clinical Neurology

http://n.neurology.org/cgi/collection/all_clinical_neurology All Genetics

http://n.neurology.org/cgi/collection/all_genetics

All Movement Disorders

http://n.neurology.org/cgi/collection/all_movement_disorders

Information about reproducing this article in parts (figures,tables) or in its entirety can be found online at:

http://www.neurology.org/about/about_the_journal\#permissions

Information about ordering reprints can be found online:

http://n.neurology.org/subscribers/advertise

Neurology ${ }^{\circledR}$ is the official journal of the American Academy of Neurology. Published continuously since 1951, it is now a weekly with 48 issues per year. Copyright (O 2019 American Academy of Neurology. All rights reserved. Print ISSN: 0028-3878. Online ISSN: 1526-632X.

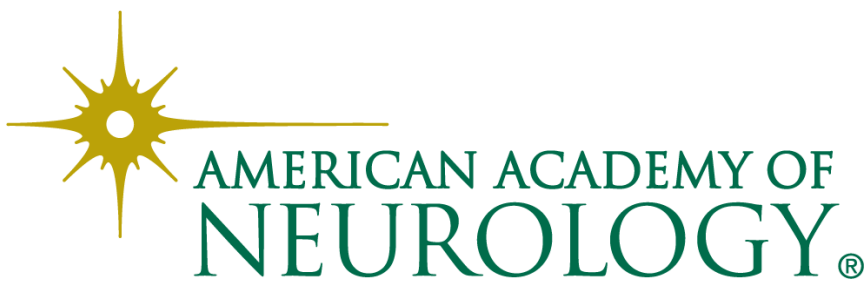

